\title{
Depicting the evolving scenario of translational-guided drug development
}

\author{
G. Argilés $\cdot$ J. Rodon $\cdot$ J. Tabernero
}

Received: 30 October 2012/ Accepted: 30 October 2012/Published online: 13 November 2012

(c) Federación de Sociedades Españolas de Oncología (FESEO) 2012

The development process of most important targeted agents currently used in oncologic clinical practice has unveiled the fact that using these types of drugs efficaciously is best supported by the availability of appropriate predictive biomarkers. Conversely, the magnitude of the effect obtained from administering such drugs to patients that respond to them on the basis of known biomarkers is likely to be diluted by concomitantly administering the drugs to an unselected patient population as well. This scenario could compromise the future of bona fide compounds due to the bias of non-selection when measuring drug benefit.

Usually this agent-biomarker matching process and its approval based on positive indications of spent takes in identifying a rational biomarker, and then the back end process of validating the assay used to measure it. In this regard, Bando and collaborators compare, in this issue of the journal, the three methods for determining KRAS mutation in colorectal carcinoma with the goal of delineating the best test for this purpose.

The history of the role of KRAS mutations in metastatic colorectal carcinoma well illustrates the diverse processes underlying predictive biomarker identification for antineoplastic targeted agents during the last 20 years, as reviewed in Table 1.

\footnotetext{
G. Argilés $(\bowtie) \cdot$ J. Rodon $\cdot$ J. Tabernero

Medical Oncology Department, Vall d'Hebron University

Hospital, Barcelona, Spain

e-mail: gargiles@vhebron.net

J. Rodon

e-mail: jrodon@vhio.net

J. Tabernero

e-mail: jtabernero@vhio.net
}

Mutations in codons 12, 13, 61 and 126 of the KRAS protein were accepted as determinants of resistance to treatment with anti-epidermal growth factor receptor monoclonal antibodies (anti-EGFR MoAbs) in colorectal carcinoma, years after cetuximab was first granted approval for its treatment by regulatory agencies [1,2]. At that time, the development of anti-EGFR MoAbs was focused on EGFR amplification and overexpression as the primary characteristics for selecting patients for clinical trials [3, 4]. The discovery of the role of KRAS mutations redirected the development plans for these compounds, causing some ongoing clinical trials to be amended to exclude patients with KRAS activating mutations. When this new approach was instituted, clinical endpoints were successfully met, leading these drugs to broad approval in many clinical settings of colorectal carcinoma.

This example and other successes resulting from the correct implementation of translational research data in clinical trials have shifted the panorama of drug development (Table 1). Accordingly, we have witnessed in the last decade clinical and translational research in oncology being brought closer together in a process intended to develop in parallel the best targeted agents and to align them with the best predictive biomarkers to guide their use starting with in early phases of the drug development process. This rapprochement bridging the gap between research and clinical application has modified both arenas. Such alignment is shown not only in the increasing number of research institutes linked to health care facilities but also by the changes that phase 1 clinical trial units have undergone [5].

In keeping with this trend, the search for biomarkers of drug effect (pharmacodynamic markers) and identification of potential target subpopulations of patients that, until recently, has occurred only in the last phases of drug 
Table 1 Approved biomarker-drug pairs used on normal clinical practice in oncology

\begin{tabular}{|c|c|c|c|c|}
\hline Biomarker & Assay & Drug & Target & Disease \\
\hline HER2 expression/amplification & IHC, FISH & Trastuzumab & HER2 & $\begin{array}{l}\text { Breast carcinoma and gastric } \\
\text { adenocarcinoma }\end{array}$ \\
\hline Estrogen receptor expression & IHC & Tamoxifen, aromatase inhibitors & Estrogen receptors & Breast cancer \\
\hline EGFR mutation codon 19,21 & PCR, Sanger & Gefinitinib, erlotinib & EGFR & Lung adenocarcinoma \\
\hline$K R A S$ mutation codons $12,13,61$ & Sanger, PCR & Cetuximab, panitumumab & EGFR & Colorectal carcinoma \\
\hline$B R A F$ mutation $\mathrm{V} 600 \mathrm{E}$ & PCR & Vemurafenib & BRAF & Melanoma \\
\hline CKIT mutation & PCR & Imatinib & CKIT & GIST \\
\hline PDGFR mutation & PCR & Imatinib & PDGFR & GIST \\
\hline$E M L 4-A L K$ translocation & FISH & Crizotinib & ALK & Lung adenocarcinoma \\
\hline
\end{tabular}

$H E R 2$ human epidermal growth factor 2, EGFR epithelial growth factor receptor, KRAS Kirsten rat antigen sarcoma protein, $P D G F R$ plateletderived growth factor receptor, EMLA echinoderm microtubule-associated protein-like 4, ALK anaplastic lymphoma kinase, IHC immunohistochemistry, FISH fluorescence in situ hybridization, PCR polymerase chain reaction, GIST grastro-intestinal stromal tumor

development, is currently shifting to early clinical trials. Thus, phase 1 studies are progressively occupying a more central role in the development plan of new agents. Recent examples of this evolution are ALK inhibitors in non-small cell lung cancer and BRAF inhibitors in melanoma $[6,7]$. Current phase 1 clinical trials are being designed to emulate these successful outcomes, enriching the participant population according to pre-specified exploratory biomarkers to identify trends and signals that can define responding patient populations at the beginning of the early drug development process. This phenomenon requires several decisions to be made. During the trajectory of a given disease when should the patients be screened? Which genetic and epigenetic determinants should be analyzed and which technology is best suited to these purposes? In early drug development, biomarkers are explored as potential predictive markers, leaving the validation of sensitivity, specificity and reproducibility of determination assays for later stages when the relationship between biomarker and response of a given compound has already been determined.

As outlined above, we have transitioned from a clinically directed drug development approach in which responses to drugs in specific tumor types guided the next steps of experimentation to a translational guided scenario in which the predictive biomarkers and drugs are codeveloped from the beginning of drug development until drug approval [8]. However, the increasing role translational research has gained toward defining a more extensive characterization of tumors has to be balanced by potential disadvantages to cancer patients that this evaluation process may cause. Institutions dealing with advanced cancer patients must ensure that the patient remains at the center of the stake. It is important not to lose sight that the fundamental basis driving translational research and drug development is to develop better therapies for patients who are likely to respond to them. What is novel is that emerging new drugs (or revisited old drugs) are enhanced by knowledge of the molecular biology of the disease as well as the capacity to molecularly profile the tumor. These improvements in drug development are increasingly offered to cancer patients who could benefit from them. One of the risks of this approach is to transform the hospitals in basic research institutes. It is the responsibility of the physicians at the helm of these research institutions as well as individual investigators to safeguard patient care and arbitrate in favor of the best interests of the patients they serve.

Conflict of interest None.

\section{References}

1. Cunningham D, Humblet $Y$ et al (2004) Cetuximab monotherapy and cetuximab plus irinotecan in irinotecan-refractory metastatic colorectal cancer. $\mathrm{N}$ Engl J Med 351(4):337-345

2. Allegra CJ, Jessup JM et al (2009) American Society of Clinical Oncology provisional clinical opinion: testing for KRAS gene mutations in patients with metastatic colorectal carcinoma to predict response to anti-epidermal growth factor receptor monoclonal antibody therapy. J Clin Oncol 27(12):2091-2096

3. Argiles G, Dienstmann R et al (2012) Panitumumab: a summary of clinical development in colorectal cancer and future directions. Future Oncol 8(4):373-389

4. Broadbridge V, Karapetis C, Price T (2012) Cetuximab in metastatic colorectal cancer. Expert Rev Anticancer Ther 12(5):555-565

5. Rodon J, Saura C et al (2012) Molecular prescreening to select patient population in early clinical trials. Nat Rev Clin Oncol 9(6):359-366

6. Kwak E, Bang Y et al (2010) Anaplastic lymphoma kinase inhibition in nonsmall-cell lung cancer. N Engl J Med 363(18):1693-1703

7. Chapman $P$, Hauschild A et al (2011) Improved survival with vemurafenib in melanoma with BRAF V600E mutation. N Engl J Med 364(26):2507-2516

8. García V, Cassier P, de Bono J (2011) Parallel anticancer drug development and molecular stratification to qualify predictive biomarkers: dealing with obstacles hindering progress. Cancer Discov 1(3):207-212 